Vol. 5, Issue 1, January 2017

\title{
Improved VSRP using Route Table in VANET
}

\author{
Jatinder Kumar ${ }^{1}$, Vikram Mutneja ${ }^{2}$, Inderjeet Singh Gill ${ }^{3}$ \\ Research Scholar, Department of ECE, S.B.S. STC, Ferozepur (Punjab), India ${ }^{1}$ \\ Assistant Professor, Department of ECE, S.B.S. STC, Ferozepur (Punjab), India ${ }^{2,3}$
}

\begin{abstract}
Technology in day to day life is developing with very high speed and many new branches have been originated from the wireless sensor networks. The VANET is also a branch of the Wireless Sensor Networks. In VANET the information is shared between nodes through wireless communication network. As security is an essential issue in WSN, any successful attack can cause a great harm or threat for drivers as well as passengers. It can decrease the speed of the whole network and may put a great impact on the performance of the network. Routing between the moving vehicles is very challenging and quite interesting task. In this research work, the performance evaluation of the GPSR (Greedy Perimeter Stateless Routing) which is very popular Position Based Routing (PBR) in VANET is done by comparing it with other routing techniques i.e. VSRP (Vehicular Security through Reputation and Plausibility Checks) technique and the proposed improved Reputation and Plausibility Checks technique using Route table. In proposed technique, the dataflow route table is used to improve the results of its counterpart technique (VSRP) using the speed, direction and the orientation of the neighbouring vehicles. The comparison between the results of all the three techniques is done and it is concluded that the results of proposed technique are better than VSRP with the same conditions and parameters.
\end{abstract}

Keywords: Greedy Perimeter Stateless Routing (GPSR), Position Based Routing (PBR), Vehicular Security through Reputation and Plausibility Checks (VSRP), VANET and NS2.

\section{INTRODUCTION}

Due to increase in vehicles traveling on the road day-byday, the transport system is facing a number of challenges.As the number of accidents happening on the road is thereby increasing at the same rate. To control this situation it becomes essential to provide the road safety and comfort for drivers. Therefore, a new research area is developed in wireless sensor networks named as Vehicular Ad-hoc Networks (VANET). Systems like VANET play an important role in safety as well as non-safety applications [1]. Collision avoidance, Driver drowsiness prevention system, Emergency warning system, Automatic emergency braking systems are included in the safety applications. On the other side, the traffic information systems like direction changer, cooperative entertainment, toll service, Internet access falls under the Non-safety applications.[2] VANET is a subclass of the MANET.

MANET is a wireless decentralized and self-organized network of a large number of mobile nodes connected temporarily [4]. But for fast moving vehicles VANET is preferred because it is self-organized and spread network [3]-[16]. In VANET since the connection is established only for few seconds due to fast moving vehicles dynamic topology and not is being used[5]-[6]. In this research, VANET network is evaluated using popular Position Based Routing (PBR) i.e. GPSR (Greedy Perimeter Stateless Routing) and VSRP (Vehicular Security through Reputation and Plausibility Checks) techniques. The PBR does not handle the false location generation efficiently and also requires digital signatures, which needs additional infrastructure. The comparison of both the techniques is done and a new technique is proposed, which use the concept of Route table to improve the results of VSRP. Finally, the comparison is done between the three techniques and the result of proposed technique i.e. improved VSRP is found better than existing VSRP and PBR under the same conditions and with same parameters.

The performance for the different protocols is evaluated in the form of some parameters [17] i.e. Throughput, End to End Delay, Network Routing Load and the Packet Delivery Ratio:

Throughput of the network is defined as the total number of packets received per unit time at the server. Throughput is measured in bits/sec and for the improvement in the network performance, this must be increased.

End to end delay is the time taken by a packet to be transmitted through a network from source to destination. Also end to end delay is the delay of the each node to the total packets transmitted through the network.

$>$ Network routing load is used to balance the traffic without the use of complex routing across the two WAN links. When forwarding a packet, it allows the router to use multiple paths to send the packet to its destination and this must be decreased to improve the network performance.

$>$ The packet delivery ratio is the ratio of the packets delivered successfully to the destination as compared to the number of packets transmitted by the sender. To 
improve the network performance this PDR must be maximum.

This paper is organized as follows: Section 1 gives the basic information about the VANET. Section 2 describes the related work. Section 3 defines the proposed technique which is improved Vehicular Security through Reputation and Plausibility Checks (I-VSRP). Section 4 describes the results and discussions in which results are shown in the form of mentioned parameters. Section 5 defines the Conclusion and Future scope.

\section{RELATED WORK}

A lot of research and effort has been put in this area to develop the more secure routing techniques in VANET. To develop a system which is more secure, reliable and cost effective is not an easy task. A number of techniques are already introduced to achieve these goals like position based routing, reputation based system, plausibility and the sensor driven technique, cryptographic method and the combination of the reputation and the plausibility technique i.e. VSRP. Some of the related protocols in detail are discussed below:

\section{A. Position Based Routing:}

Hannes Hartenstein et al. [7] did a survey and mainly focused on the location based routing for VANETs and evaluating the GPSR which is very popular position based routing protocol. In Location based approaches with the help of on-board navigation systems, the vehicles are made aware of their geographical positions.

C. Harsch et al. [9] did a survey on secure position-based routing for VANETs. They proposed defence mechanisms, relying both on cryptographic scheme and plausibility checks technique. They used cryptographic scheme to achieve security in VANETs and concluded that their implementation and initial measurements show the proposed scheme deployable and also the security overhead is low. Plausibility checks reduce the impact of false positions on the routing operation.

\section{B. Reputation and Plausibility Check techniques:}

F. Dotzer et al. [10] mainly focused on the enhanced security method in VANET and they proposed a VARS (vehicle ad-hoc networks reputation system) technique, a completely distributed technique based on reputation system. One of the major challenge is to forward the information related to the event and the information is trusted by the receiving node.

N. Wei Lo et al. [11] mainly targeted on the message plausibility attacks problem that may result in the accidents and traffic jams. So to prevent this type of attack they develop a new model which is known as the plausibility validation network (PVN) model. In this they found a new attack that is specific to VANETs, called Illusion attack and a possible solution to address this attack is proposed through a plausibility mechanism. In this they have presented the illusion attack, a severe security threat that can manipulate a driver's behaviour in vehicular ad hoc networks. Also they concluded that in the illusion attack, a malicious attacker creates specific traffic situation and sends the fraud traffic warning messages to other drivers.

Sanjay K. Dhurandher et al.[12] designed a system on Vehicular Security through Reputation and Plausibility Checks technique which is the combination of the reputation and plausibility techniques. They present vehicular security through plausibility and reputation check techniques to describe one of the most important issues of VANET i.e. Security issue. The algorithm was simulated and tested on different scenarios and they observed it is very effective in terms of the percentage of detection of malicious nodes, number of packets sent after malicious nodes are detected, average time taken to detect the nodes which are generating false events or false information, number of packets dropped in VANET, and the number of packets received by malicious nodes. They concluded that the proposed algorithm provides security against the attacks like data aggregation, data dropping, event modification and false event generation. It performs detection of malicious nodes and also provides the isolation of malicious nodes in the network.

\section{PROPOSED TECHNIQUE: IMPROVED VSRP}

The security is a major issue in VANET because in VANET the information is shared between nodes through the wireless communication network. Any successful attack can cause a great harm or threat for drivers as well as passengers [13]-[14]. It can decrease the speed of the whole network and may put a great impact on the performance of the network. So designing a highly secure network becomes an open research area for researchers and developers. Few challenges that come in the way of researchers are like the use of fast cryptographic algorithms, authentication of nodes from external and internal attacks, key distribution for encryption and decryption of a message and high mobility of nodes.[15] In this research work a technique is proposed and is called as improved Vehicular Security through Reputation and Plausibility Checks (I-VSRP). This technique uses the Route table concept which is more effective than position based routing and VSRP. In this concept, it uses four main things i.e. Route table, Greedy approach, trust value and the next hop.

Methodology: First of all, the system of the source or sender node checks for the destination node with the greedy approach. If the two nodes are present near to the sender node then the sender node will check the route table. The route table will tell the trust value of both the nodes by calculating their position, orientation, speed and some other factors also. In this way, the sender node will get the trust value of both the other nodes and send the data to the node with more trust value for once. But for 


\section{International Journal of Innovative Research in Electrical, Electronics, Instrumentation and Control Engineering \\ ISO 3297:2007 Certified}

Vol. 5, Issue 1, January 2017

sending the data again the next hop works. Again the whole process is repeated in search for the node with the higher trust value and again the greedy approach is followed. Now the data is again transferred by using the concept of route table.

\section{RESULTS AND DISCUSSIONS}

In Position Based Routing (PBR), only the greedy approach is followed and no check for the security of the networks has been followed. Vehicular Security through Reputation and Plausibility Check (VSRP) technique provides the security, but with the limitation that the sender node does not have any route table to compare the trust values of two destination nodes.

\begin{tabular}{|c|}
\hline$\times($ code.tcl \\
\hline Throughput \\
\hline Packet Delivery Ratio \\
\hline End to End Delay \\
\hline Network Routing Load \\
\hline Cancel
\end{tabular}

Fig 1: GUI pushbuttons in NS2

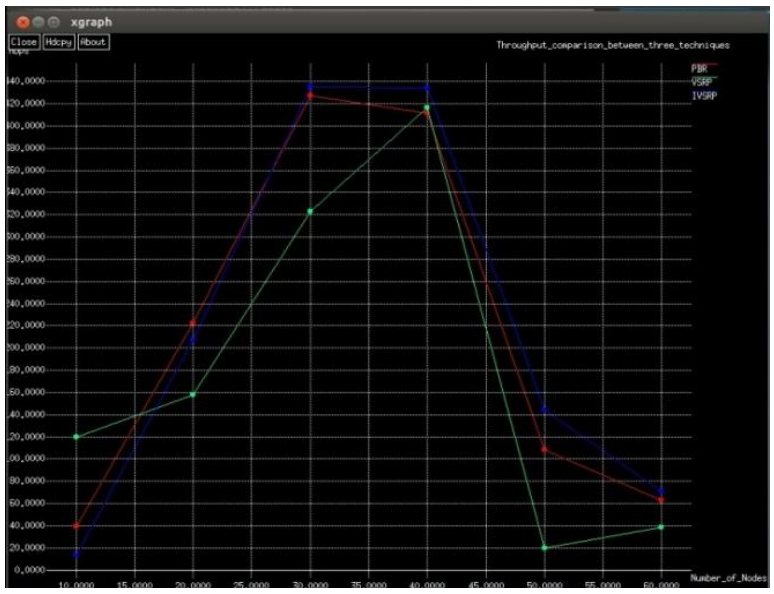

Fig 2: Throughput comparison between three techniques

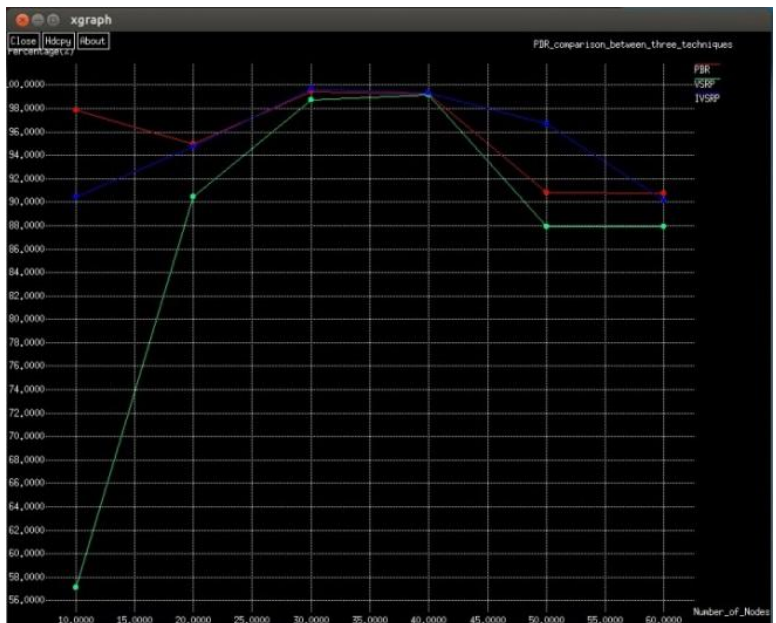

Fig 3: PDR comparison between three techniques
Hence it follows mainly the greedy approach and sends the data to the node with positive trust value and also which is near to it. These limitations are removed in the proposed technique - Improved VSRP. This technique is found better than the mentioned techniques in terms of some parameters.

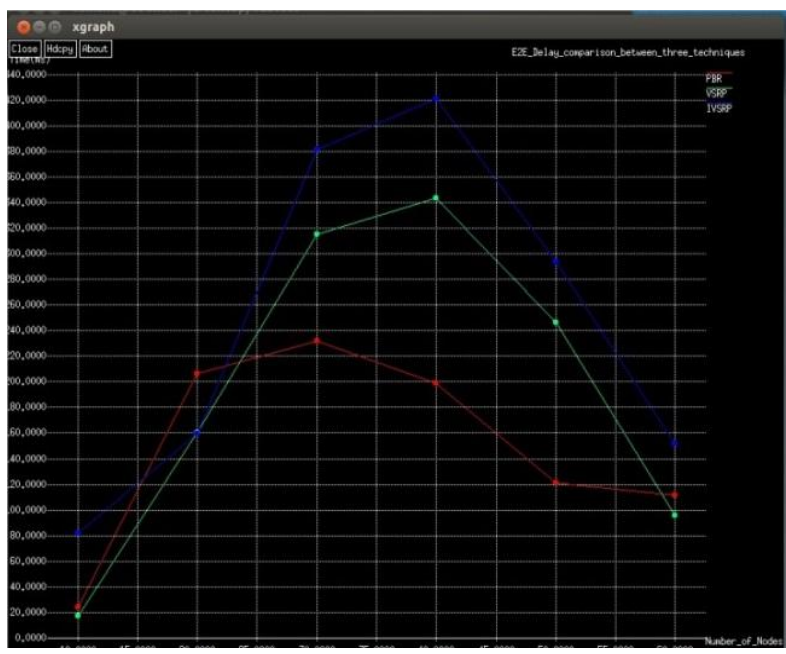

Fig 4: E2E Delay comparison between the three techniques

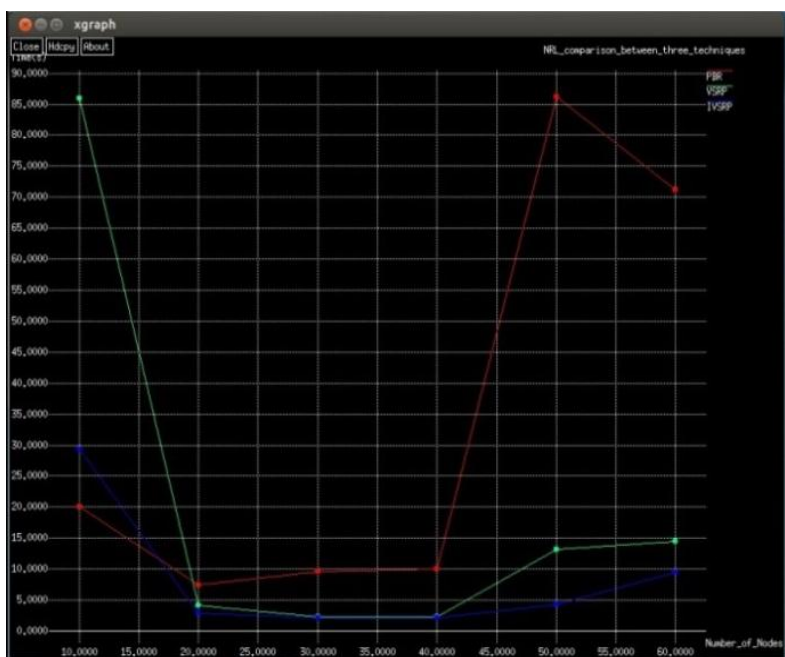

Fig 5: NRL comparison between three techniques

Analysis: In the above figures, four results are shown. The Fig. 1 shows the Graphical User Interface push buttons in NS2. The results are calculated for 10 nodes, 20 nodes, 30 nodes, 40 nodes, 50 nodes and 60 nodes in every graph. The Fig. 2 shows the Throughput comparison between the three techniques. The value of the throughput is required to be high. The Fig. 3 shows the Packet delivery ratio comparison between the three techniques. The value of the Packet delivery ratio must be high. The Fig. 4 shows the End to end delay comparison between the three techniques. The value of the End to end delay is required to be low. The Fig. 5 shows the Network routing load comparison between the three techniques. The value of the Network routing load must be low. 


\section{International Journal of Innovative Research in Electrical, Electronics, Instrumentation and Control Engineering ISO 3297:2007 Certified}

Vol. 5, Issue 1, January 2017

The proposed work has been simulated using the two types of tools namely SUMO and Network Simulator 2 and a Graphical User Interface has been developed to facilitate the user to open a graph for displaying the results and do the comparison. User can increase or decrease the number of buttons and get the graphs displayed as per requirement After simulation, it is observed that the output values of the basic Position based routing are better sometimes because it is considered to be an ideal case. In PBR, it is considered that there is no attack and so no security technique is being used.

In VSRP, the attacks are taken into consideration and hence they can be detected and removed also. It is not taken as the ideal case like the PBR. So the results are diminished in the VSRP than the PBR. The third is the proposed technique- improved VSRP which is used to enhance the efficiency of the system. It is found that the results of this technique are better specifically between nodes 30 and 40 in terms of Throughput, Packet Delivery Ratio, and Network Routing Load. However, the results in terms of End to End delay are found to be less effective. But it can be compensated for the sake of security and later on improvements may be made for future research.

\section{CONCLUSION AND FUTURE SCOPE}

In this research, an overview of VANET is discussed focusing on various research issues and challenges that come while implementing VANET in vehicular telematics. It is found that in Position Based Routing (PBR) only the greedy approach is followed and there is no check for the security of the networks. Vehicular Security through Reputation and Plausibility Check (VSRP) technique provides the security, but with the limitation that the sender node does not have any route table to compare the trust values of two destination nodes. These limitations are removed in the technique being analysed and it is found better than its counterpart techniques in terms of different parameters.

To evaluate the realistic simulation environment for vehicles, Network Simulator 2 (NS2) and Simulation for Urban Mobility (SUMO) platforms have been used. The performance of the system is measured by using the parameters like Network Throughput, Packet Delivery Ratio (PDR), Network Routing Load (NRL) and Average End to End Delay (E2E Delay) and the results and it is observed that the said technique gives better performance in terms of few parameters.

In future, we are preparing to work further on Improved VSRP technique so as to further improve the performance parameters like the Network Routing Load and End to end delay. The values of the Throughput and the Packet Delivery Ratio also requirethe further improvement. It is also necessary to detect the exact number of malicious nodes in a short time period and to eliminate them with the decrease in the complexity of the network system.

\section{REFERENCES}

[1] SheraliZeadalli, Ray Hunt, Yuh-Shyan Chen ,Angela Irwin and Aamir Hassan "Vehicular ad hoc networks (VANETS) status, results and challenges", Telecommunication Systems, DOI 10.1007/s11235-010-9400-5, @ Springer Science + Business Media, LLC 2010.

[2] Md. HumayunKabir, "Research Issues on Vehicular Ad hoc Network", International Journal of Engineering Trends and Technology (IJETT) - Volume 6, Number 4, pp. 174-179, Dec 2013.

[3] S. Swapna Kumar, "Vehicular Ad-hoc Network", International Journal of Computer, Electrical, Automation, Control and Information Engineering, Vol. 8, No: 4, pp. 590-593, 2014.

[4] JeroenHoebeke, Ingrid Moerman, Bart Dhoedt and Piet Demeester "An Overview of Mobile Ad Hoc Network: Applications and Challenges", Department of Information Technology (INTEC), Ghent University - IMEC vzw, SintPietersnieuwstraat 41, B-9000 Ghent, Belgium. Session-4, pp. 60-66.

[5] Yasser Toor And Paul Muhlethaler, InriaAnisLaouiti, Get/Int Arnaud De La Fortelle, Ecole Des Mines, "Vehicles Ad hoc networks: Applications and Related Technical Issues", IEEE Communications Surveys \& Tutorials, 3rd Quarter 2008, Volume 10, No. 3, pp. 74-88.

[6] DaxinTian, KavehShafiee and Victor C. M. Leung "Position Based Directional Vehicular Routing",Global Telecommunications Conference, 2009, IEEE pp. 1-6, DOI: 10.1109/GLOCOM.2009.

[7] Hannes Hartenstein, Dieter Vollmer and HolgerFubler, "LocationBased Routing for Vehicular Ad-hoc Networks", MOBICOM'02, September 23-28, 2002, Atlanta, Georgia, USA.

[8] M. Mauve, J. Widmer and H. Hartstein, "A Survey on Position Based Routing on Mobile Ad-hoc Networks", IEEE Networks, Volume-15, Issue-6, pp. 30-39, Nov-Dec 2001.

[9] C. Harsch, A. Festag, and P. Papadimitratos, "Secure positionbased routing for VANETs", in Proc. Veh. Technol. Conf., 2007, pp. 26-30.

[10] F. Dotzer, L. Fischer, and P. Magiera, "VARS: A vehicle ad-hoc network reputation system," in Proc. $6^{\text {th }}$ IEEE Int. Symp. World Wireless Mobile Multimedia Netw., June. 2005, pp. 454-456.

[11] N.W. Lo and H.C. Tsai, "Illusion attack on VANET applicationsA message plausibility problem", in Proc. 2nd IEEE Workshop Autom.Network Appl., Nov. 2007, pp. 1-8.

[12] Sanjay K. Dhurandher, Mohammad S. Obaidat and AmritJaiswal, "Vehicular Security through Reputation and Plausibility Checks", IEEE System Journal, Vol. 8, No. 2, June 2014.

[13] Maxim Raya and Jean Pierre Hubaux, "The Security of VANETs", VANET'05, September 2, 2005, Cologne, Germany. (ACM: 1595931414 /05/0009), pp. 93-94.

[14] Ram Shringar Raw, Manish Kumar and Nanhay Singh, "Security Challenges, Issues and their Solutions for VANET", International Journal of Network Security \& its Applications, Vol.5, No.5, September 2013.

[15] Surmukh Singh and Sunil Agrawal, "VANET Routing Protocols: Issues and Challenges", in the proceedings of 2014 RAECS UIET Punjab University Chandigarh, 06-08 March, 2014.

[16] Jatinder Kumar and Inderjeet Singh Gill, "Research Issues, Applications and Challenges in Vehicular Ad-hoc Networks: A survey", National Conference on Communication, Computing and Systems, pp: 53-57, Aug 2015

[17] Jatinder Kumar, Vikram Mutneja and Inderjeet Singh Gill, "Behavior of Position based routing in VANET", International Journal of Computer Applications (0975-8887) Volume 145- No. 1, July 2016. 